\title{
REGIME FLUVIALE DEL TEVERE E VARIAZIONE DEL LITORALE DELTIZIO DAL RINASCIMENTO AI NOSTRI GIORNI
}

\author{
PIERO BELLOTTI \\ piero.bellotti@gmail.com \\ SAPIENZA UNIVERSITÀ DI ROMA \\ http://dx.doi.org/10.25267/Riparia_sup.2018.11.07
}

\section{Generalità sul bacino del fiume Tevere}

Il bacino del Fiume Tevere è il maggiore dell'Italia peninsulare e si estende per poco più di $17.000 \mathrm{~km}^{2}$. Il fiume scorre per $405 \mathrm{~km}$ dall'Appennino toscoemiliano al Tirreno con una portata media prossima a $230 \mathrm{~m}^{3} / \mathrm{s}$. La minima portata misurata è stata di circa $70 \mathrm{~m}^{3} / \mathrm{s}$ mentre la massima di $2750 \mathrm{~m}^{3} / \mathrm{s}$. In assenza di ghiacciai nel bacino, le portate sono determinate quasi esclusivamente dalle piogge, sono massime nel semestre autunno-inverno.

La parte più orientale del bacino è costituita da potenti successioni carbonatiche meso-cenozoiche che consentono un'ampia riserva idrica garantendo, attraverso i bacini affluenti dei fiumi Nera e Aniene, un flusso di base di circa 100 $\mathrm{m}^{3} / \mathrm{s}$. Nella restante parte del bacino i sedimenti clastici e vulcanici, che hanno una media e bassa permeabilità, favoriscono la variabilità delle portate e costituiscono la maggior parte del carico solido fluviale.

\section{Il regime fluviale del Tevere nel tempo}

Per il periodo romano le fonti indicano 34 principali eventi di piena in poco più di 800 anni dei quali ben 24 verificatisi tra il I secolo a.C e il II d.C. (Carcani, 1875 ; Bersani e Bencivenga, 2001). Ricerche geo-archeologiche confermano che nel periodo di massima frequenza si verificò la crisi di funzionalità del porto di Ostia (Goiran et al., 2014) e le massime difficoltà nell'ingresso alla foce del Tevere.

Tra VI e XI secolo vengono riportati solo 14 eventi ad indicare il periodo di minore attività fluviale dell'alto Medioevo. I dati del periodo romano e soprattutto alto medievale hanno un certo grado di incertezza essendo soggetti alla qualità e alla capacità dei cronisti del tempo.

I dati divengono via via più completi e affidabili dal 1180 quando oltre ad una più precisa collocazione temporale (data esatta) si inizia a indicare il livello raggiunto dalle acque nella città di Roma (fig. 1). Le altezze raggiunte durante le piene non sono registrate in modo metodico ma indicate su lapidi sparse nelle parti basse della città e su due colonne presenti nel porto fluviale di Ripetta. Il valore dell'altezza idrometrica raggiunta in Roma dalle acque delle piene verificatesi tra il 1180 e il 1870 fu definito nel 1871 quando nel porto di Ripetta fu stabilito un idrometro per la misura diretta e continua dell'altezza idrometrica e a tale idrometro furono riportate le altezze indicate nelle diverse lapidi. 

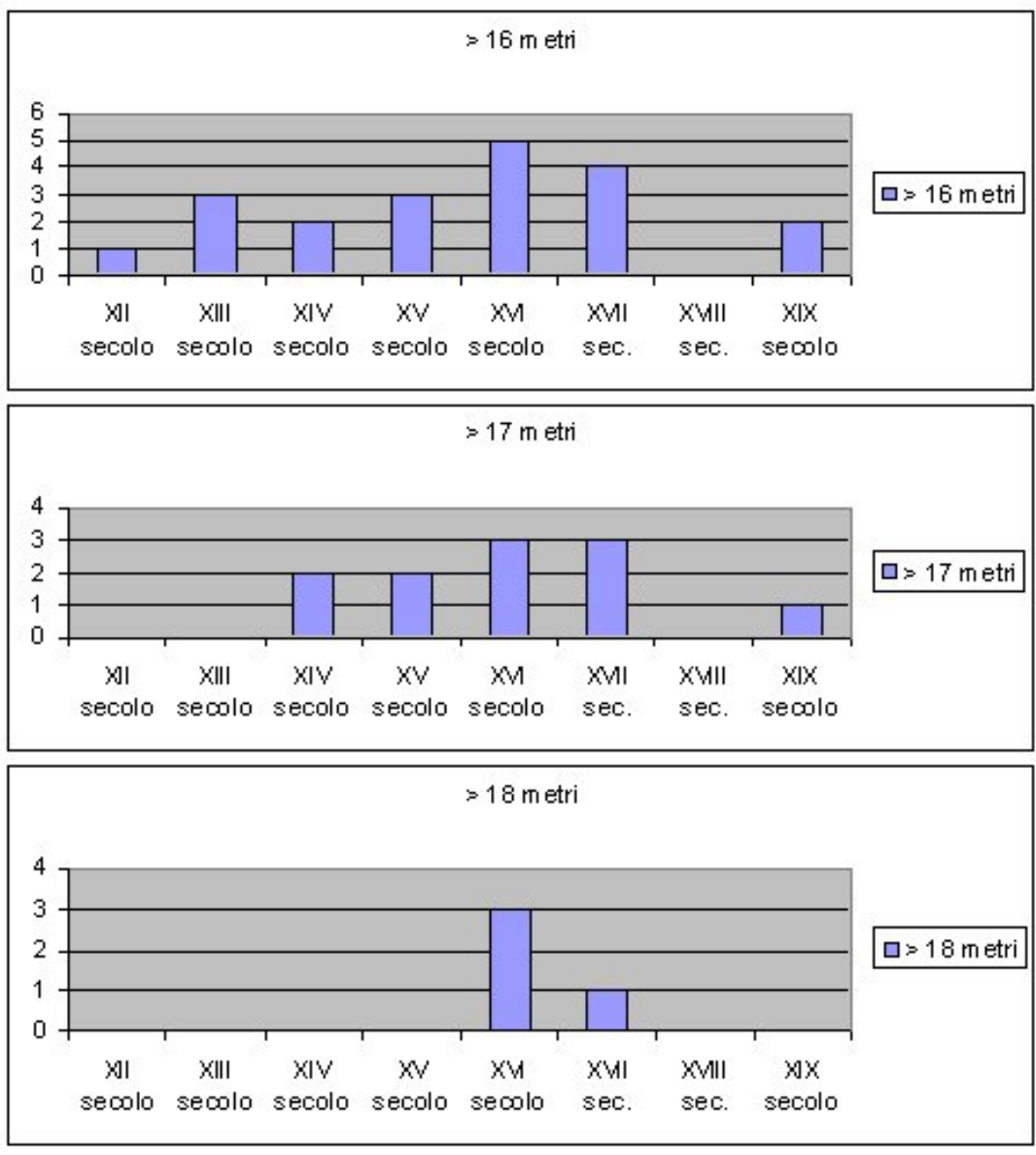

Fig. 1. Numero delle piene del F. Tevere tra i secoli XII e XIX che hanno superato a Roma le altezze idrometriche di 16, 17 e 18 metri.

Nel XV secolo vengono segnalati, sia pure con pochi dettagli, ben 15 eventi di cui due superarono con certezza l'altezza idrometrica di 17 metri, mentre nel XVI e XVII secolo si segnala la maggiore frequenza delle piene con maggiore altezza al colmo (Martino e Belati 1980). Per tre volte vennero superati i 18 metri nel XVI secolo e una nel XVII. Una tale frequenza e intensità degli eventi di piena risulta coincidente con il periodo fresco-umido detto Piccola Età Glaciale che interessò tutto l'emisfero boreale. Si consideri che la piena del 1557, oltre ad inondare Roma, mutò il corso del Tevere in prossimità di Ostia e fu pressoché coincidente con le alluvioni di Firenze (F. Arno), Grosseto (F. Ombrone) e Palermo (Fossa della Garofala, Fiumetto e F. Oreto) indicando un evento meteorologico che in alcuni giorni investì gran parte dell'Italia. Dopo il 1606 non è più stata raggiunta l'altezza idrometrica di 18 metri mentre i 17 metri furono superati solo nelle piene del 1637, 1660 e 1870. La relazione tra la Piccola Età Glaciale che inizia nel XIV secolo e si esaurisce nella seconda metà del XIX appare evidente.

Dal 1871 inizia la registrazione continua dell'altezza idrometrica all'idrometro di Ripetta e fino al 1920 solo in due casi venne superata l'altezza di 16 metri. Per alcuni di tali eventi si è calcolata a posteriori la portata relativa che è risultata massima nella piena del 2 dicembre del 1900 con $3100 \mathrm{~m}^{3} / \mathrm{s}$.

Il riassetto urbanistico dei primi anni del XX secolo confinò il tratto urbano il Tevere tra due muraglioni, la stazione di misura fu modificata e dal 1921 iniziarono le misure dirette della portata. Da allora solo nel 1937 fu superata l'altezza di 16 m con 
$2750 \mathrm{~m}^{3} / \mathrm{s}$. Dal 1947 la presenza di alcune dighe modera i picchi di piena e l'altezza idrometrica non ha più raggiunto i 14 metri.

Sulla base dei dati pluviometrici e idrometrici del periodo 1921-2000 si è determinato per il Tevere un tempo di concentrazione di circa 62 ore. Si è inoltre osservato che le piene $>1500 \mathrm{~m}^{3} / \mathrm{s}$ (eccezionali ed estreme) si verificano quando le precipitazioni si protraggono per 3-4 giorni superando il valore di $50 \mathrm{~mm}$ cumulati e mediati su tutto il bacino. Occasionalmente anche piogge di soli 2 giorni (intense sulla parte più impermeabile del bacino) possono produrre piene eccezionali. L'effetto delle precipitazioni è comunque funzione dello stato di saturazione dei terreni al momento dell'inizio dell'evento piovoso.

I caratteri salienti del regime fluviale del Tevere dal Rinascimento ad oggi sembrano dunque essere i seguenti :

- Le piene del Tevere sono comuni tra ottobre e marzo ; occasionali in maggio e settembre. Gli eventi sono determinati essenzialmente dalle piogge ;

- Gli eventi piovosi della durata di 3-4 giorni sull'intero bacino sono responsabili dei maggiori eventi di piena che occasionalmente possono essere indotti anche da 2 giorni di piogge intense.

- La massima frequenza delle piene con $\mathrm{h}>17$ metri all'idrometro di Ripetta si è avuta tra XIV e XVII secolo.

- Le massime altezze idrometriche sono registrate nel XVI e all'inizio del XVII secolo. Il valore massimo assoluto è stato di $19.58 \mathrm{~m}$ nell'evento del 1598 . Il valore di portata in questi eventi può essere stimata tra 3500 e $4000 \mathrm{~m}^{3} / \mathrm{s}$

- Il regime fluviale sembra essere mutato nel tempo concordemente con le variazioni climatiche plurisecolari. Le piene risultano più frequenti e imponenti in coincidenza con l'acme della Piccola Età Glaciale per poi progressivamente diminuire la loro intensità. Il solo XVIII secolo appare estraneo a tale andamento.

\section{Considerazioni sul trasporto torbido del Tevere}

Le osservazioni sul trasporto solido in sospensione hanno mostrato che questo varia concordemente con la portata ma non in modo lineare. Con portate inferiori a $350 \mathrm{~m}^{3} / \mathrm{s}$ il trasporto in sospensione è trascurabile. Esso diviene importante con portate comprese tra $350 \mathrm{~m}^{3} / \mathrm{s}$ e $700 \mathrm{~m}^{3} / \mathrm{s}$, oltre tale valore è trasportata in sospensione anche l'intera frazione sabbiosa (Bersani e Piotti, 1994).

Misure dirette e continue del trasporto torbido sono state effettuate alla fine del XIX secolo dal 1873 al 1878 e, nel XX secolo tra il 1932 e il 1973. Dal 1973 le misure dirette sono state discontinue. L'esame dei dati evidenzia un continuo decremento dei valori. Il valor medio dei primi sei anni di registrazioni si attesta a circa 10.6 milioni di tonnellate per anno ; questo valore riguarda un bacino ancora in uno stato quasi naturale con scarsa influenza delle opere umane. Il valor medio calcolato tra il 1932 e il 1947 risulta di poco inferiore a 7.5 milioni di tonnellate per anno. In questo periodo nel bacino si era già prodotta l'importante arginatura del Tevere all'interno e a valle della città di Roma ed erano stati costruiti i primi bacini idroelettrici su alcuni affluenti (F. Turano e F. Salto). Tra il 1947 e il 1962 furono costruiti due importanti bacini idroelettrici sul corso del Tevere; quello di Castel Giubileo, al limite settentrionale di Roma (il più vicino alla foce), e quello maggiore di Corbara. Il valor medio del trasporto in sospensione dopo il 1962 si attesta intorno a 1.5 milioni di tonnellate per anno e dai primi anni ' 90 del secolo scorso il valore 
annuale del trasporto torbido è stato generalmente inferiore al milione di tonnellate per anno.

La diminuzione del valor medio tra la fine del XIX secolo e la prima metà del XX si deve in parte al miglioramento climatico dovuto alla fine della Piccola Età Glaciale e ai primi bacini idroelettrici costruiti sui fiumi Salto e Turano. L'ulteriore riduzione del trasporto torbido avvenuta nella seconda metà del XX secolo è interamente da imputare all'azione dell'uomo esercitata con la costruzione di bacini idroelettrici e il prelievo di sedimento nell'alveo tiberino.

\section{Evoluzione della linea di riva nell'area deltizia}

L'evoluzione della linea di riva in prossimità delle foci è ben documentata da diverse cartografie storiche (fig. 2). La carta di Eufrosino della Volpaia (1547), che riporta il meandro di Ostia prima dell'inizio del suo abbandono dovuto alla piena del 1557, mostra una linea di riva ancora scarsamente cuspidata. Tale lineamento morfologico indica che negli anni precedenti non si era verificato un rapido avanzamento delle foci. In realtà è noto che la foce minore nel periodo tardo medievale ebbe una funzionalità discontinua e che nello stesso periodo le grandi piene non furono frequenti. L'acquerello di Horatio Torriani (1603) disegnato durante l'acme alluvionale della Piccola Età Glaciale, verificatosi come detto tra il XVI e la prima metà del XVII secolo, riporta alcuni interessanti lineamenti. Il corso principale del Tevere ha ormai assunto l'attuale andamento dopo il completo abbandono del meandro di Ostia e alla foce principale è riportato, in destra della foce principale, un prominente apice deltizio. Si può dedurre che a seguito delle maggiori piene, in precedenza citate, la foce principale sia rapidamente avanzata in mare. La carta di Chiesa e Gambarini (1744) fornisce una eccellente immagine della costa deltizia caratterizzata da una prominente cuspide alla foce principale e a una evidente progradazione di tutta la linea di riva che rispetto alla carta del Torriani risulta regolarizzata. Anche la carta di Amenduni (1884) conferma l'aspetto cuspidato del delta tiberino con la linea di riva leggermente più regolare e avanzata in mare rispetto alla cartografia di Chiesa e Gambarini.
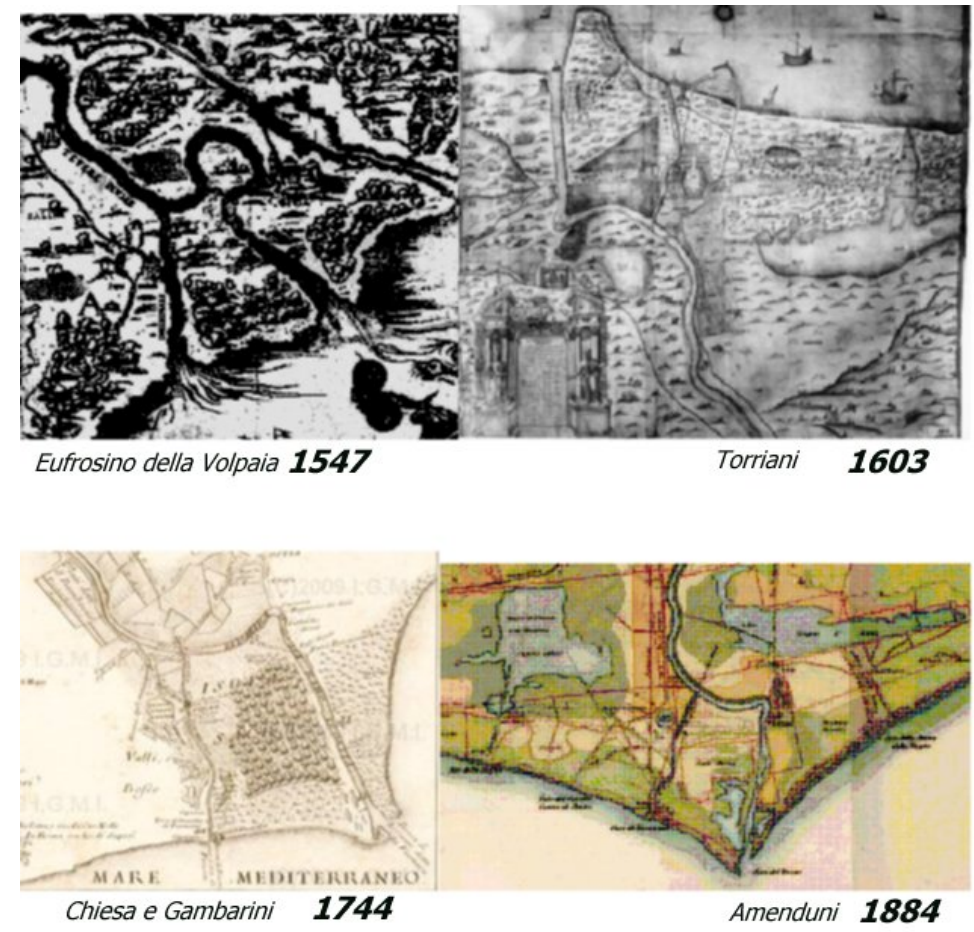

Fig. 2. Carte storiche del delta del F. Tevere citate nel testo. 
L'esame della cartografia storica, unitamente all'analisi geometrica dei cordoni costieri e alle torri di guardia costruite alle foci via via che queste avanzavano in mare, mostra che la progradazione è avvenuta con una fase particolarmente intensa intorno al XVI secolo e, pur con locali e temporanee fasi di arretramento, è proseguita in modo evidente fino al XVIII secolo per poi rallentare e arrestarsi quasi completamente all'inizio del XX. Nel complesso, tra il Rinascimento e l'inizio del XX secolo, si è prodotta una progradazione dell'apice deltizio di circa $3 \mathrm{~km}$.

La tendenza evolutiva sembra essersi invertita solo alla metà del XX secolo quando la linea di riva inizia ad arretrare. Tuttavia il confronto tra i rilievi batimetrici del 1883 e del 1939 (fig. 3) pone in evidenza che l'erosione dell'apice deltizio inizia già nella prima parte del XX secolo. La situazione del 1883, quando il Tevere aveva un trasporto torbido superiore 10 milioni di tonnellate annue, era caratterizzata da un'ampia spiaggia poco acclive soprattutto nella parte sommersa. La distanza delle batimetriche di -5 e -10 metri dalla foce principale, misurata sul prolungamento dell'asse del canale di Fiumara Grande, era rispettivamente 1300 e 4400 metri. Nel 1939, con un trasporto torbido inferiore a 8 milioni di tonnellate annue, tali distanze si erano rispettivamente ridotte a 300 e 1500 metri con un conseguente notevole incremento dell'acclività del fondale. La linea di riva tuttavia risultava in tale intervallo di tempo sostanzialmente stabile. L'ulteriore riduzione del trasporto solido successivo alla costruzione delle dighe lungo il corso del Tevere ha indotto intensi arretramenti della linea di riva nella seconda metà del XX secolo.

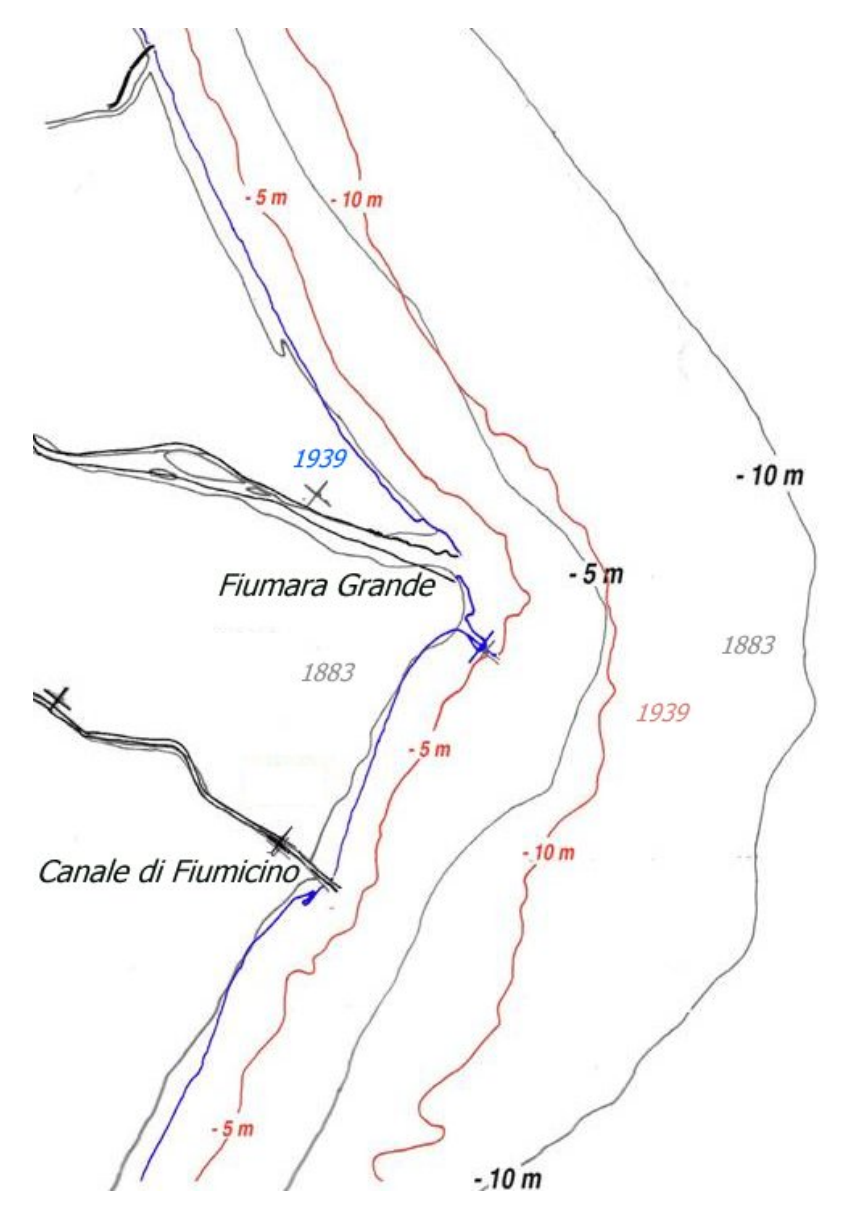

Fig. 3. Sourapposizione dei rilievi cartografico-batimetrici, semplificati, dell'area di foce del F. Tevere tratti dalle carte dell'I.I.M. degli anni 1883 e 1939. 


\section{Considerazioni finali}

I dati per stabilire una relazione numerica tra il regime fluviale e l'evoluzione della costa sono decisamente insufficienti. Tuttavia si può osservare che l'area emersa del delta aumentava ancora con portate torbide maggiori di 10 milioni di tonnellate annue e che appariva stabile con portate prossime a 7.5 milioni di tonnellate annue, quando però l'erosione del delta sommerso era già in atto. Portate torbide inferiori hanno generato l'arretramento della linea di riva in tutto l'apice deltizio. Tale erosione procederebbe ancora se non si fosse provveduto a stabilizzare la linea di riva mediante opere di difesa rigide (essenzialmente barriere frangiflutti) o interventi di ripascimento artificiale più o meno protetti.

I valori della portata torbida rilevati alla fine del XIX secolo, pur rappresentando i massimi valori misurati, dovevano essere inferiori a quelli delle fasi in cui si ebbe il massimo tasso di progradazione. Quei valori possono soltanto essere stimati e si può supporre che possano essere stati, durante le piene del XVI e XVII secolo, 3 o 4 volte maggiori di quelli misurati alla fine del XIX secolo. E' difficile dire anche quale sia stata la piovosità nel bacino del Tevere che generò quei valori del trasporto torbido. Al di là di valori parossistici che dovettero produrre le piene eccezionali del XVI/XVII secolo, fu probabilmente sufficiente che la piovosità fosse tale da consentire un frequente superamento della portata soglia $\left(700 \mathrm{~m}^{3} / \mathrm{s}\right)$ che produce il massimo rifornimento di sabbia al litorale. Non va comunque trascurato che la quantità di sedimento disponibile dipende anche dall'uso che in epoche diverse si è fatto del territorio.

Per quanto il Tevere sia stato storicamente una importante via d'acqua, il suo regime ne ha sempre resa complessa la navigabilità commerciale e ancor oggi crea problemi a quella turistica.

\section{Bibliografia}

P. Bersani, M. Bencivenga, Le piene del Tevere a Roma dal V secolo a. C. all'anno 2000, Roma, 2001, URL: https://speleology.files.wordpress.com/2012/ 06/piene tevere roma.pdf.

P. Bersani, A. PiotTI, «Correlazioni tra portata liquida e trasporto solido del fiume Tevere a Roma ». Idrotecnica 2, 1994, p. 69-78.

M. CARCANI, Il Tevere e le sue inondazioni dalle origini di Roma ai giorni nostri. Roma, Tipografia Romana, 1875.

V. Di Martino, M. Belati, Qui arrivò il Tevere: le inondazioni del Tevere nelle testimonianze e nei ricordi storici (lapidi, idrometri, cronache, immagini), Roma, 1980.

J.-P. Goiran, F. Salomon, I. Mazzini, J.P. Bravard, E. Pleuger, C. VitTori, "Geoarchaeology confirms location of the ancient harbour basin of Ostia (Italy)», Journal of Archaeological Science 41, p. 389-398. 